\title{
Condensed-Phase Ethanol Conversion to Higher Alcohols over Bimetallic Catalysts
}

Iman Nezam, Jason Zak, and Dennis J. Miller

Department of Chemical Engineering and Materials Science

Michigan State University

East Lansing, Michigan 48824

\section{Supplementary Information}

Table S1. Nickel and bimetallic catalyst bulk compositions on a mass and molar basis. The balance is $\gamma-\mathrm{Al}_{2} \mathrm{O}_{3}$ in all cases. Bimetallic catalysts are prepared in noted mass ratios by adding a second metal to $\mathrm{Ni}(\mathrm{C})\left(8.0 \mathrm{wt} \% \mathrm{Ni} / 9.0 \mathrm{wt} \% \mathrm{La}_{2} \mathrm{O}_{3} / \gamma-\mathrm{Al}_{2} \mathrm{O}_{3}\right)$ catalyst. Ni-Cu bimetallic is made in two different metal mass ratios.

\begin{tabular}{|c|c|c|c|c|c|c|c|c|c|c|}
\hline \multirow{2}{*}{ Catalyst } & \multicolumn{5}{|c|}{ Mass \% } & \multicolumn{5}{|c|}{ Mole \% } \\
\hline & $\mathrm{Ni} / \mathrm{M}$ & $\mathbf{N i}$ & $\mathbf{M}$ & $\mathbf{N i}+\mathbf{M}$ & $\mathbf{L a}_{2} \mathrm{O}_{3}$ & $\mathbf{N i} / \mathbf{M}$ & $\mathbf{N i}$ & $\mathbf{M}$ & $\mathbf{N i}+\mathbf{M}$ & $\mathrm{La}_{2} \mathrm{O}_{3}$ \\
\hline $\begin{array}{c}\mathrm{Ni}(\mathrm{A}) \\
8.0 \mathrm{wt} \% \mathrm{Ni} / 4.5 \mathrm{wt} \% \mathrm{La}_{2} \mathrm{O}_{3} / \gamma-\mathrm{Al}_{2} \mathrm{O}_{3}\end{array}$ & - & 8.0 & - & - & 4.5 & - & 14.1 & - & - & 1.4 \\
\hline $\begin{array}{c}\mathrm{Ni}(\mathrm{B}) \\
8.0 \mathrm{wt} \% \mathrm{Ni} / 9.0 \mathrm{wt} \% \mathrm{La}_{2} \mathrm{O}_{3} / \gamma-\mathrm{Al}_{2} \mathrm{O}_{3}\end{array}$ & - & 8.0 & - & - & 9.0 & - & 13.9 & - & - & 2.8 \\
\hline $\begin{array}{c}\mathrm{Ni}(\mathrm{C}) \\
8.0 \mathrm{wt} \% \mathrm{Ni} / 9.0 \mathrm{wt} \% \mathrm{La}_{2} \mathrm{O}_{3} / \gamma-\mathrm{Al}_{2} \mathrm{O}_{3}\end{array}$ & - & 8.0 & - & - & 9.0 & - & 13.9 & - & - & 2.8 \\
\hline $\begin{array}{c}\mathrm{Ni}(\mathrm{D}) \\
8.0 \mathrm{wt} \% \mathrm{Ni} / 9.0 \mathrm{wt} \% \mathrm{La}_{2} \mathrm{O}_{3} / \gamma-\mathrm{Al}_{2} \mathrm{O}_{3}\end{array}$ & - & 8.0 & - & - & 9.0 & - & 13.9 & - & - & 2.8 \\
\hline $\begin{array}{c}\mathrm{Ni(E)} \\
1.0 \mathrm{wt} \% \mathrm{Ni} / 9.0 \mathrm{wt} \% \mathrm{La}_{2} \mathrm{O}_{3} / \gamma-\mathrm{Al}_{2} \mathrm{O}_{3}\end{array}$ & - & 1.0 & - & - & 9.0 & - & 2.0 & - & - & 3.4 \\
\hline $\begin{array}{c}11.5 \mathrm{wt} \%(\mathrm{Ni} / \mathrm{Cu} 2 / 1) / 8.7 \mathrm{wt} \% \\
\mathrm{La}_{2} \mathrm{O}_{3} / \gamma-\mathrm{Al}_{2} \mathrm{O}_{3}\end{array}$ & 2 & 7.7 & 3.8 & 11.5 & 8.7 & 2.2 & 13.1 & 6.0 & 19.1 & 2.7 \\
\hline $\begin{array}{c}8.4 \mathrm{wt} \%(\mathrm{Ni} / \mathrm{Cu} 20 / 1) / 9.0 \mathrm{wt} \% \\
\mathrm{La}_{2} \mathrm{O}_{3} / \gamma-\mathrm{Al}_{2} \mathrm{O}_{3} \\
\end{array}$ & 20 & 8.0 & 0.4 & 8.4 & 9.0 & 22.7 & 13.6 & 0.6 & 14.2 & 2.7 \\
\hline $\begin{array}{c}11.5 \mathrm{wt} \%(\mathrm{Ni} / \mathrm{Co} 2 / 1) / 8.7 \mathrm{wt} \% \\
\mathrm{La}_{2} \mathrm{O}_{3} / \gamma-\mathrm{Al}_{2} \mathrm{O}_{3}\end{array}$ & 2 & 7.7 & 3.8 & 11.5 & 8.7 & 2.0 & 13.0 & 6.5 & 19.5 & 2.6 \\
\hline $\begin{array}{c}9.7 \mathrm{wt} \%(\mathrm{Ni} / \mathrm{Pd} 4 / 1) / 8.8 \mathrm{wt} \% \\
\mathrm{La}_{2} \mathrm{O}_{3} / \gamma-\mathrm{Al}_{2} \mathrm{O}_{3}\end{array}$ & 4 & 7.8 & 1.9 & 9.7 & 8.8 & 7.2 & 13.7 & 1.9 & 15.6 & 2.8 \\
\hline $\begin{array}{c}9.7 \mathrm{wt} \%(\mathrm{Ni} / \mathrm{Pt} 4 / 1) / 8.8 \mathrm{wt} \% \\
\mathrm{La}_{2} \mathrm{O}_{3} / \gamma-\mathrm{Al}_{2} \mathrm{O}_{3}\end{array}$ & 4 & 7.8 & 1.9 & 9.7 & 8.8 & 13.8 & 13.8 & 1.0 & 14.8 & 2.8 \\
\hline $\begin{array}{c}11.5 \mathrm{wt} \%(\mathrm{Ni} / / \mathrm{Fe} 2 / 1) / 8.7 \mathrm{wt} \% \\
\mathrm{La}_{2} \mathrm{O}_{3} / \gamma-\mathrm{Al}_{2} \mathrm{O}_{3}\end{array}$ & 2 & 7.7 & 3.8 & 11.5 & 8.7 & 1.9 & 13.0 & 6.8 & 19.8 & 2.6 \\
\hline $\begin{array}{c}11.5 \mathrm{wt} \%(\mathrm{Ni} / \mathrm{Mo} 2 / 1) / 8.7 \mathrm{wt} \% \\
\mathrm{La}_{2} \mathrm{O}_{3} / \gamma-\mathrm{Al}_{2} \mathrm{O}_{3}\end{array}$ & 2 & 7.7 & 3.8 & 11.5 & 8.7 & 3.4 & 13.4 & 4.0 & 17.4 & 2.7 \\
\hline
\end{tabular}




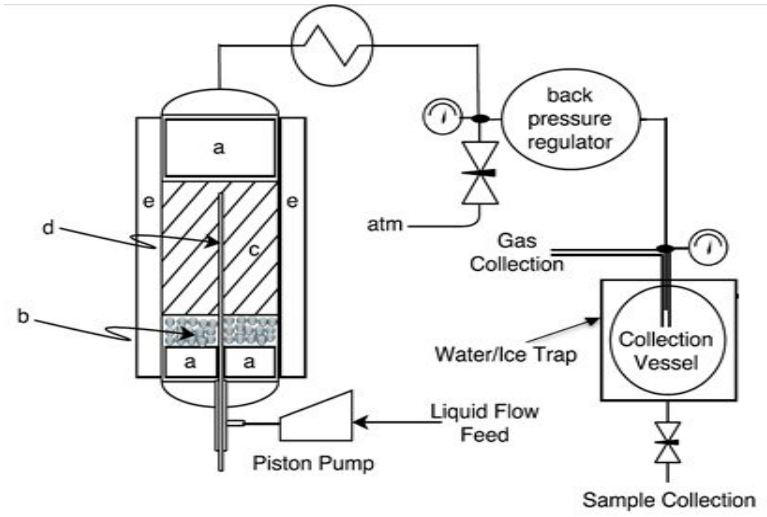

Figure S1. Continuous flow reactor for ethanol conversion. a - stainless steel rods to reduce dead volume; $\mathrm{b}$ - SiC packing to preheat feed; $\mathrm{c}$ - catalyst bed; $\mathrm{d}$ - thermowell; $\mathrm{e}$ - oil jacket. 


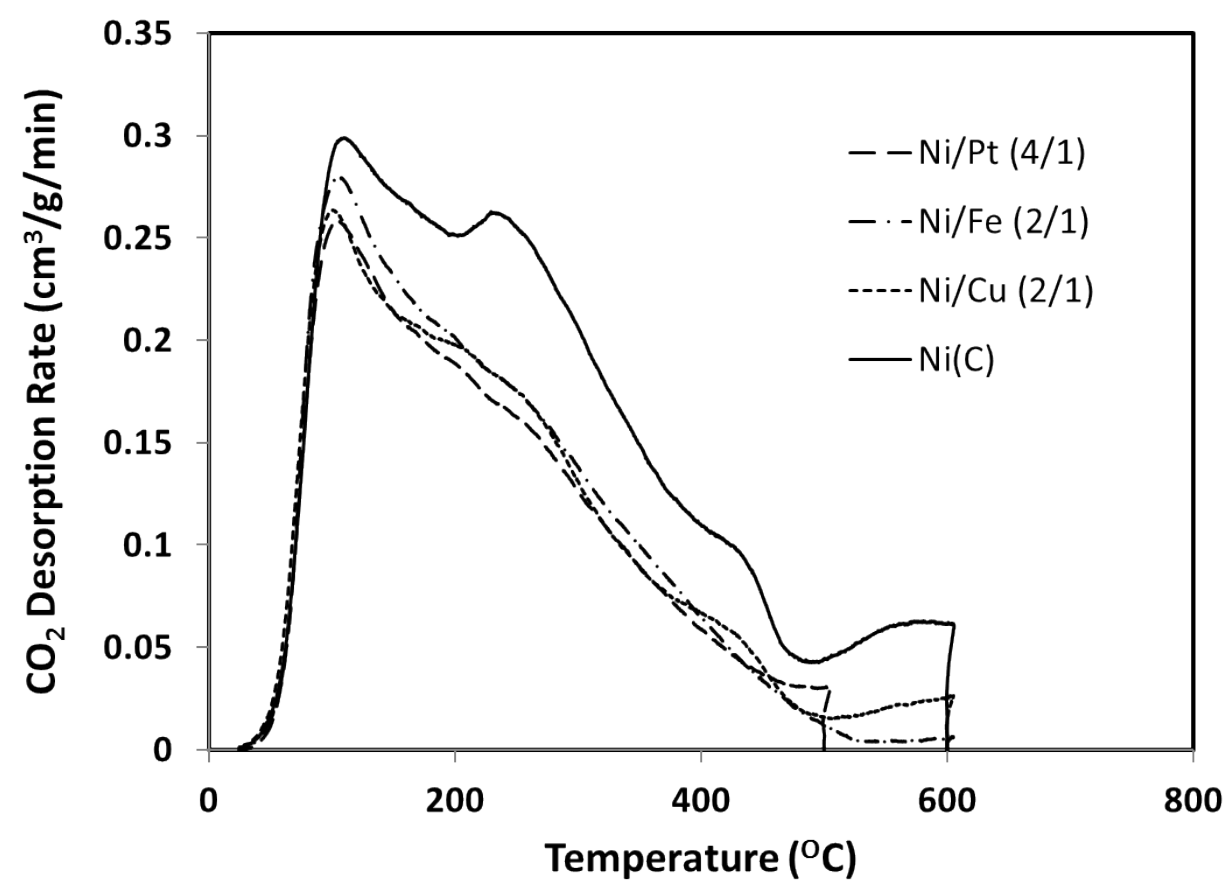

Figure S2. Temperature programmed desorption profiles of $\mathrm{CO}_{2}$ from selected catalysts. Heating rate $10^{\circ} \mathrm{C} / \mathrm{min}$ from $25^{\circ} \mathrm{C}$ to $\left.600^{\circ} \mathrm{C} \mathrm{(Ni/Pt} \mathrm{heated} \mathrm{to} 500{ }^{\circ} \mathrm{C}\right)$ in $50 \mathrm{ml} / \mathrm{min}$ He carrier gas.

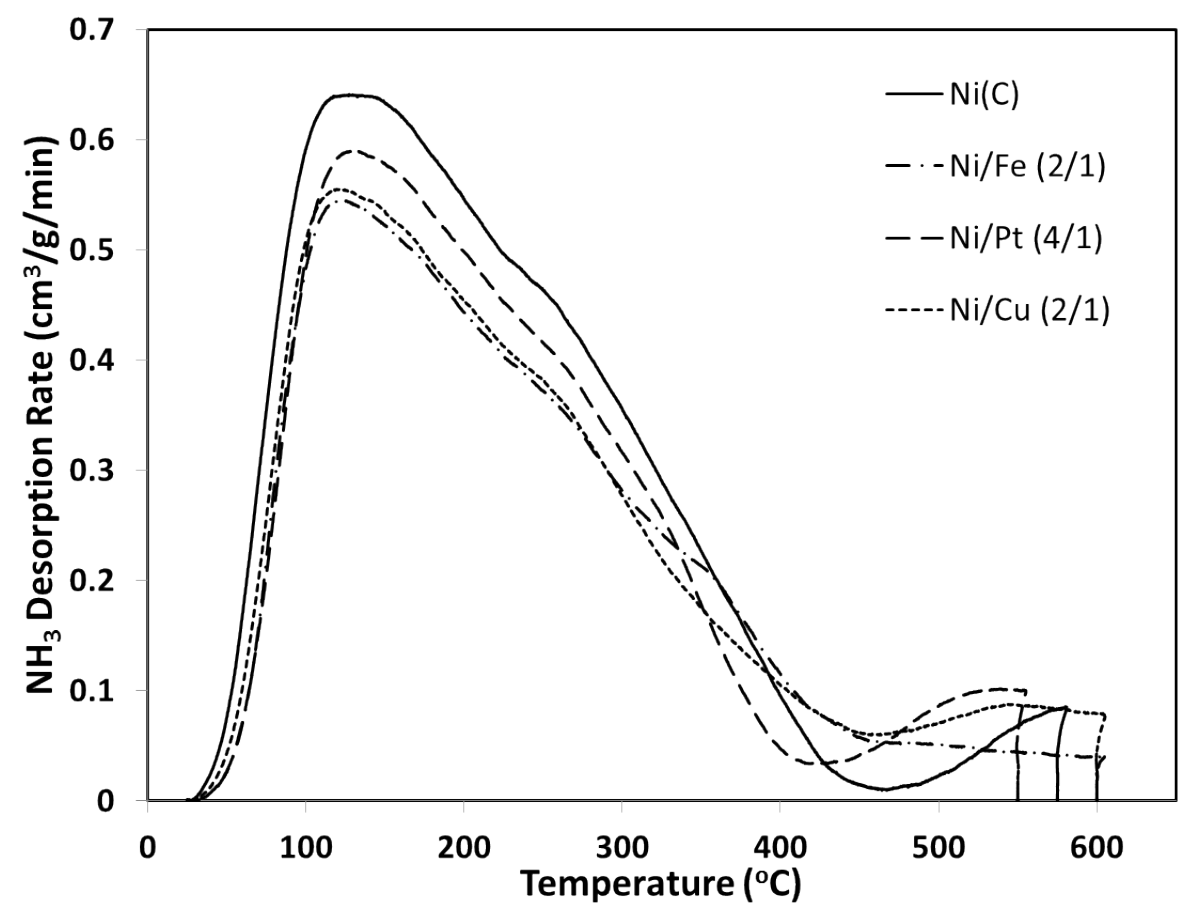

Figure S3. Temperature programmed desorption profiles of $\mathrm{NH}_{3}$ from selected catalysts. Heating rate $10^{\circ} \mathrm{C} / \mathrm{min}$ from $25^{\circ} \mathrm{C}$ to $600{ }^{\circ} \mathrm{C} \mathrm{(Ni/Pt} \mathrm{heated} \mathrm{to} 550^{\circ} \mathrm{C}$ ) in $50 \mathrm{ml} / \mathrm{min}$ He carrier gas. 


\section{Approach to Equilibrium of Condensed-Phase Ethanol Dehydrogenation}

To examine more closely the role of ethanol dehydrogenation in condensation to $n$-butanol, the equilibrium constant $\left(\mathrm{K}_{\mathrm{e}}\right)$ for ethanol dehydrogenation (Reaction R1 below) in the liquid phase was calculated using standard heats of formation $\left(\Delta \mathrm{H}_{\mathrm{f}, \text { liq }}\right)$ and entropies $\left(\mathrm{S}_{\mathrm{o}}\right)$ for liquid ethanol and acetaldehyde, and gaseous hydrogen taken from the NIST Chemistry Webbook. Results at different temperatures are given in Table S2 below.

To obtain an estimate of the approach to equilibrium of ethanol dehydrogenation, the actual concentrations and mole fractions of ethanol, acetaldehyde, and hydrogen in the outlet of the continuous reactor were calculated for all experiments with $\mathrm{Ni}(\mathrm{A})$ catalyst, and the ratio of $\mathrm{y}_{\mathrm{Ac}}{ }^{*} \mathrm{y}_{\mathrm{H} 2} / \mathrm{y}_{\mathrm{EtOH}}$ was determined. It is noted that the concentration of $\mathrm{H}_{2}$ in gaseous products was determined by GC in every experiment; however, the quantity of $\mathrm{H}_{2}$ formed is not reported in Tables S5 or S8 because only carbon-containing gaseous products are included there. The experimental mole fraction ratio was then compared to the calculated equilibrium constant $\mathrm{K}_{\mathrm{e}}$ as a measure of the approach of ethanol dehydrogenation to equilibrium. This is admittedly an inexact comparison, because non-idealities in the near-critical, condensed phase reaction solution are ignored. However, since the activity coefficient of acetaldehyde in ethanol is less than 1.0 at low acetaldehyde concentration, ${ }^{\mathrm{S} 1}$ the actual departure from equilibrium will be greater than that predicted by this calculation. Results are given in Table S2; they show that at most the experimental mole fraction ratio is $28 \%$ of the calculated equilibrium constant for the reaction, so the reaction is substantially departed from equilibrium. The same calculations were carried out for all other Ni catalysts; the approach to equilibrium of other catalysts is substantially smaller than for $\mathrm{Ni}(\mathrm{A})$, which has the highest metal dispersion and thus the highest activity.

These results support the hypothesis that ethanol dehydrogenation is not at equilibrium during reaction, and thus can be the rate limiting step of ethanol conversion to $n$-butanol. They also provide evidence that it is not necessary to explicitly include the reverse acetaldehyde hydrogenation reaction in the rate expression.

Table S2. Calculation of Approach to Equilibrium for Ethanol Dehydrogenation

\begin{tabular}{|c|c|c|c|c|c|c|c|}
\hline Exp \# & $\mathrm{T}\left({ }^{\circ} \mathrm{C}\right)$ & Calc. $\mathrm{K}_{\mathrm{e}}$ & $\mathrm{y}_{\mathrm{EtOH}, \exp }$ & $\mathrm{y}_{\text {Ac,exp }}$ & $\mathrm{y}_{\mathrm{H} 2, \exp }$ & $\begin{array}{c}\text { Exp Ratio } \\
\mathrm{y}_{\mathrm{Ac}} \mathrm{y}_{\mathrm{H} 2} / \mathrm{y}_{\mathrm{EtOH}}\end{array}$ & Exp Ratio/K \\
\hline $28-4$ & 170 & $1.6 \mathrm{E}-5$ & 0.93 & 0.0020 & 0.0020 & $4.3 \mathrm{E}-6$ & 0.27 \\
\hline $28-1$ & 190 & $4.1 \mathrm{E}-5$ & 0.88 & 0.0039 & 0.0013 & $5.8 \mathrm{E}-6$ & 0.14 \\
\hline $48-2$ & 210 & $9.7 \mathrm{E}-5$ & 0.54 & 0.0069 & 0.0021 & $2.7 \mathrm{E}-5$ & 0.28 \\
\hline $29-3$ & 210 & $9.7 \mathrm{E}-5$ & 0.65 & 0.0076 & 0.0023 & $2.7 \mathrm{E}-5$ & 0.27 \\
\hline $29-2$ & 210 & $9.7 \mathrm{E}-5$ & 0.71 & 0.0075 & 0.0023 & $2.5 \mathrm{E}-5$ & 0.26 \\
\hline $29-2$ & 210 & $9.7 \mathrm{E}-5$ & 0.75 & 0.0066 & 0.0026 & $2.3 \mathrm{E}-5$ & 0.24 \\
\hline $48-1$ & 210 & $9.7 \mathrm{E}-5$ & 0.74 & 0.0074 & 0.0022 & $2.2 \mathrm{E}-5$ & 0.22 \\
\hline $48-3$ & 210 & $9.7 \mathrm{E}-5$ & 0.73 & 0.0072 & 0.0018 & $1.8 \mathrm{E}-5$ & 0.18 \\
\hline $28-3$ & 210 & $9.7 \mathrm{E}-5$ & 0.75 & 0.0075 & 0.0019 & $1.9 \mathrm{E}-5$ & 0.20 \\
\hline
\end{tabular}

S1. D’Avila, S.G.; Silva, R.S.F. Isothermal Vapor-Liquid Equilibrium Data by Total Pressure Method. J. Chem. Eng. Data 1970, 15(3), 421-424. 


\section{Kinetic Model for Ethanol Conversion}

Development of Kinetic Model: The indirect mechanism for ethanol condensation to higher alcohols involves several steps, the first two of which are

Ethanol (E) dehydrogenation to acetaldehyde (Ac): $\quad \mathrm{E}=\mathrm{Ac}+\mathrm{H}_{2}$ Acetaldehyde (Ac) condensation to crotonaldehyde (Ca): $2 \mathrm{Ac} \rightarrow \mathrm{Ca}+\mathrm{H}_{2} \mathrm{O}$

As seen above, ethanol dehydrogenation is a catalytic, reversible reaction that occurs on the metal surface sites, while acetaldehyde condensation is an irreversible reaction that takes place on the basic sites of the catalyst.

$$
\begin{gathered}
r_{1}=-r_{E}=k_{1}\left(C_{E}-\frac{C_{A c} C_{H 2}}{K_{e} C_{s o l n}}\right) \\
r_{2}=r_{C 4}=k_{2} C_{A c}^{2}
\end{gathered}
$$

If ethanol condensation to $n$-butanol is the only reaction taking place, then $C_{A c}=C_{H 2}$, since each mole of hydrogen liberated by ethanol dehydrogenation is consumed in hydrogenation of the subsequent $\mathrm{C} 4$ condensation product. Although this equality does not in reality hold, as evidenced by the experimental concentrations in Table S2 above, it is a useful approximation for development of this kinetic model that focuses on the condensation reaction.

At steady state, the rates of R1 and R2 are related to each other by $r_{1}=2 r_{2}$. Applying this equality of rates and solving for $C_{A C}^{2}$ gives the following:

$$
C_{A C}^{2}=\frac{k_{1} C_{E}}{\left(2 k_{2}+{ }^{1} 1 / K_{e} C_{\text {soln }}\right)}
$$

Inserting this expression into $r_{2}$ and rearranging gives a final expression for the rate of ethanol consumption.

$$
-r_{E}=r_{1}=2 r_{2}=\frac{C_{E}}{\left(\frac{1}{k_{1}}+\frac{1}{2 k_{2} K_{e} C_{\text {soln }}}\right)}
$$

This expression includes rate constants for the first two steps of the indirect Guerbet mechanism for reaction of ethanol to the $\mathrm{C} 4$ product, and the equilibrium constant for ethanol dehydrogenation. It simplifies if one of the two reaction steps is rate limiting, but remains first order in ethanol regardless of which step is rate limiting.

Ethanol conversion in the flow reactor can therefore be characterized by first order kinetics:

$$
-\frac{1 \mathrm{dC}_{\mathrm{E}}}{\rho_{\text {cat }} \mathrm{d \tau}}=-\mathrm{r}_{\mathrm{E}}=\mathrm{k}_{\mathrm{C}} \mathrm{C}_{\mathrm{E}}
$$

Where $\mathrm{C}_{\mathrm{E}} \quad=$ ethanol concentration $\left(\mathrm{kmol} / \mathrm{m}^{3}\right.$ solution $)$ 
$\mathrm{k}_{\mathrm{C}}=$ overall rate constant $\left(\mathrm{m}^{3}\right.$ solution $/ \mathrm{kg}$ cat $\left./ \mathrm{min}\right)$

$\rho_{\text {cat }}=$ catalyst bulk density in reactor $\left(920 \mathrm{~kg}\right.$ catalyst $/ \mathrm{m}^{3}$ reactor $)$

$\tau=$ superficial residence time $\left(\mathrm{m}^{3}\right.$ reactor $\cdot \mathrm{min} / \mathrm{m}^{3}$ solution $)=\rho_{\mathrm{EtOH}} * 60 / \rho_{\text {cat }} / \mathrm{WHSV}\left(\mathrm{h}^{-1}\right)$

$\rho_{\mathrm{EtOH}}=$ ethanol mass density at reaction temperature $\left(\mathrm{kg} / \mathrm{m}^{3}\right)$

Evaluation of Mass Transport Resistances: The influence of intraparticle mass transport on ethanol conversion for the $\mathrm{Ni}(\mathrm{A})$ catalyst at $230^{\circ} \mathrm{C}$, for which reaction is most rapid, can be evaluated via calculation of the observable modulus $\eta \varphi^{2}$ at the reactor inlet.

$$
\eta \varphi^{2}=\frac{-r_{E} L^{2}}{D_{e} C_{E o}}
$$

Where $-r_{E}=$ observed rate of ethanol consumption $\left(\mathrm{kmol} / \mathrm{m}^{3} \mathrm{cat} / \mathrm{sec}\right)$

$L=$ normalized catalyst particle size $=\mathrm{D} / 6 ; \mathrm{D}=1.6 \times 10^{-3} \mathrm{~m}$

$D_{e}=$ effective condensed phase diffusivity of ethanol in $n$-butanol, $=3 \times 10^{-9} \mathrm{~m}^{2} / \mathrm{sec}$

$C_{E o}=$ Inlet bulk concentration of ethanol at $230{ }^{\circ} \mathrm{C}$ and $100 \mathrm{bar},=13.3 \mathrm{kmol} / \mathrm{m}^{3}$

The calculated value of the observable modulus $\eta \varphi^{2}$ at the reactor inlet for $\mathrm{Ni}(\mathrm{A})$ catalyst at 230 ${ }^{\circ} \mathrm{C}$ is 0.02 , a value sufficiently low to indicate that intraparticle mass transfer limitations do not affect reaction rates at the experimental conditions of this work.

Calculation of Rate Constant: Integrating Eq. (S5) to give Eq. (S6) below allows calculation of $\mathrm{k}_{\mathrm{C}}$ for ethanol conversion $(\mathrm{x})$ at each temperature represented in Figure 3 and at each space velocity in Figure S4 below.

$$
-\ln (1-x)=\mathrm{k}_{\mathrm{C}} \rho_{\text {cat }} \tau
$$

Calculated values of $\mathrm{k}_{\mathrm{C}}$ from experimental data are plotted on a logarithmic scale against reciprocal absolute temperature in Figure S5. This Arrhenius plot gives an activation energy for ethanol conversion rate over $\mathrm{Ni}(\mathrm{A})$ catalyst of $82 \mathrm{~kJ} / \mathrm{mol}$, and a pre-exponential factor of 5230 $\mathrm{m}^{3} / \mathrm{kg}$ cat $/ \mathrm{min}$.

Applying the parameters for $\mathrm{k}_{\mathrm{C}}$ from Figure S5 allows the outlet conversion of ethanol to be predicted for data at different temperatures (Figure 3 in text) and at different WHSV (Figure S4). The predicted values of outlet ethanol conversion, shown as open circles in Figure S4 and Figure 3 , show that the first order kinetic model adequately describes ethanol conversion at these conditions. 


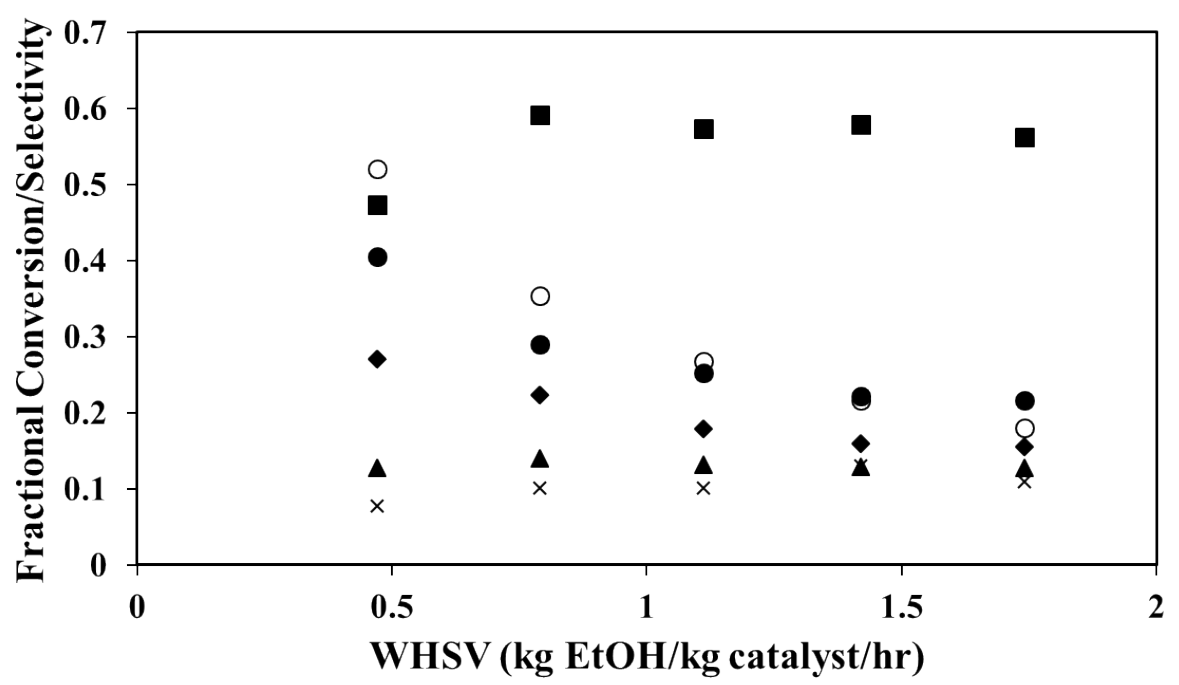

Figure S4. Ethanol conversion and product selectivity vs. weight hourly speed velocity (WHSV) for $\mathrm{Ni}(\mathrm{A})$ catalyst $\left(8.0 \mathrm{wt} \% \mathrm{Ni} / 4.5 \mathrm{wt} \% \mathrm{La}_{2} \mathrm{O}_{3} / \gamma-\mathrm{Al}_{2} \mathrm{O}_{3}\right)$ at $\mathrm{T}=210^{\circ} \mathrm{C}$. Experiment: $(\bullet)$-ethanol

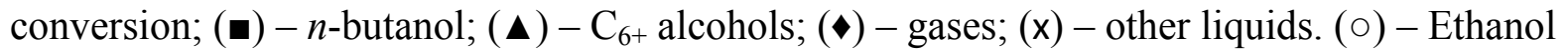
conversion predicted by first order kinetic model.

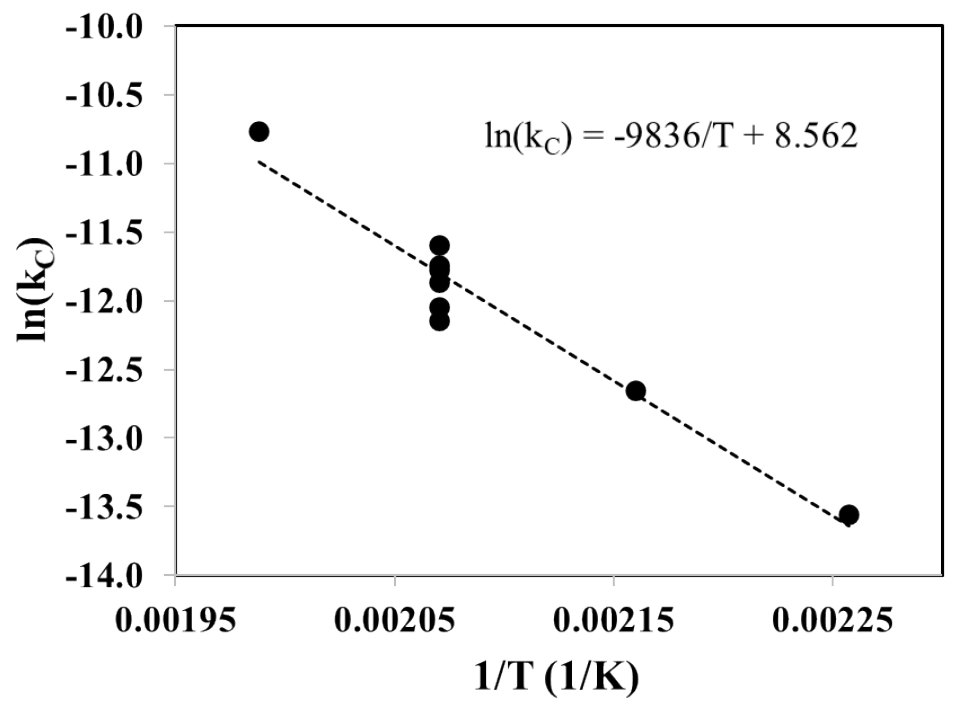

Figure S5. Arrhenius plot for first order kinetic model of ethanol conversion over Ni(A) catalyst $\left(8.0 \mathrm{wt} \% \mathrm{Ni} / 4.5 \mathrm{wt} \% \mathrm{La}_{2} \mathrm{O}_{3} / \gamma-\mathrm{Al}_{2} \mathrm{O}_{3}\right)$. Rate constant $\mathrm{k}_{\mathrm{C}}$ in $\left(\mathrm{m}^{3}\right.$ solution $/ \mathrm{kg}$ cat $\left./ \mathrm{min}\right)$. 
Table S3. Experimental conditions and results for nickel catalysts

\begin{tabular}{|c|c|c|c|c|c|c|c|c|}
\hline \multirow[b]{2}{*}{ Exp \# } & \multirow[b]{2}{*}{ Feed } & \multirow{2}{*}{$\begin{array}{c}\mathrm{T} \\
\left({ }^{\circ} \mathrm{C}\right)\end{array}$} & \multirow{2}{*}{$\begin{array}{c}\text { WHSV } \\
\left(\mathrm{h}^{-1}\right)\end{array}$} & \multirow{2}{*}{$\begin{array}{c}\text { Ethanol } \\
\text { Conv } \\
(\%)\end{array}$} & \multicolumn{4}{|c|}{ Selectivity (\%) } \\
\hline & & & & & $\begin{array}{c}n- \\
\mathrm{BuOH}\end{array}$ & $\mathrm{C}_{6+} \mathrm{OH}$ & Gas & $\begin{array}{l}\text { Liquid } \\
\text { byprod }\end{array}$ \\
\hline \multicolumn{9}{|c|}{ Promoted Support: $9.0 \mathrm{wt} \% \mathrm{La}_{2} \mathrm{O}_{3} / \gamma-\mathrm{Al}_{2} \mathrm{O}_{3}$} \\
\hline $116-2$ & Ethanol & 210 & 1.42 & 0.8 & 20 & 0.3 & - & 71 \\
\hline $116-1$ & Ethanol & 230 & 1.42 & 1.3 & 17 & 0.0 & - & 69 \\
\hline $116-3$ & Ethanol & 250 & 1.42 & 2.4 & 13 & 0.3 & - & 77 \\
\hline \multicolumn{9}{|c|}{$\mathrm{Ni}(\mathrm{A}): 8.0 \mathrm{wt} \% \mathrm{Ni} / 4.5 \mathrm{wt} \% \mathrm{La}_{2} \mathrm{O}_{3} / \gamma-\mathrm{Al}_{2} \mathrm{O}_{3}$} \\
\hline $28-4$ & Ethanol & 170 & 1.42 & 4.2 & 27 & 0.4 & 20 & 75 \\
\hline $28-1$ & Ethanol & 190 & 1.42 & 10 & 55 & 6.8 & 11 & 27 \\
\hline $48-2$ & Ethanol & 210 & 0.47 & 41 & 47 & 13 & 27 & 7.7 \\
\hline $29-3$ & Ethanol & 210 & 0.79 & 29 & 59 & 14 & 22 & 10 \\
\hline $29-2$ & Ethanol & 210 & 1.11 & 26 & 57 & 13 & 18 & 10 \\
\hline $28-2$ & Ethanol & 210 & 1.42 & 22 & 58 & 13 & 16 & 13 \\
\hline $29-1$ & Ethanol & 210 & 1.42 & 21 & 61 & 14 & 17 & 12 \\
\hline $48-1$ & Ethanol & 210 & 1.42 & 24 & 56 & 13 & 16 & 11 \\
\hline $54-1$ & $\begin{array}{c}\text { Ethanol }+0.7 \mathrm{wt} \% \\
\text { acetaldehyde }\end{array}$ & 210 & 1.42 & 24 & 57 & 17 & 15 & 7.3 \\
\hline $56-1$ & $\begin{array}{l}\text { Ethanol }+0.4 \mathrm{wt} \% \text { ethyl } \\
\text { acetate }\end{array}$ & 210 & 1.42 & 22 & 54 & 14 & 17 & 13 \\
\hline $58-1$ & Butanol & 210 & 1.46 & 16 & - & 31 & 19 & 32 \\
\hline $65-1$ & Ethanol $+4 w t \%$ water & 210 & 1.46 & 17 & 54 & 8.4 & 25 & 9.4 \\
\hline $48-3$ & Ethanol & 210 & 1.74 & 22 & 56 & 13 & 15 & 11 \\
\hline $28-3$ & Ethanol & 230 & 1.42 & 42 & 45 & 13 & 28 & 9.4 \\
\hline \multicolumn{9}{|c|}{$\mathrm{Ni}(\mathrm{B}): 8.0 \mathrm{wt} \% \mathrm{Ni} / 9.0 \mathrm{wt} \% \mathrm{La}_{2} \mathrm{O}_{3} / \gamma-\mathrm{Al}_{2} \mathrm{O}_{3}$} \\
\hline $66-1$ & Ethanol & 210 & 1.42 & 28 & 52 & 15 & 19 & 10 \\
\hline \multicolumn{9}{|c|}{$\mathrm{Ni}(\mathrm{C}): 8.0 \mathrm{wt} \% \mathrm{Ni} / 9.0 \mathrm{wt} \% \mathrm{La}_{2} \mathrm{O}_{3} / \gamma-\mathrm{Al}_{2} \mathrm{O}_{3}$} \\
\hline 83-1 & Ethanol & 210 & 0.79 & 11 & 59 & 16 & 10 & 11 \\
\hline $83-2$ & Ethanol & 210 & 1.42 & 8.2 & 60 & 13 & 8.6 & 14 \\
\hline $83-3$ & Ethanol & 210 & 2.06 & 7.2 & 59 & 11 & 8.0 & 16 \\
\hline $84-1$ & Ethanol & 230 & 0.79 & 24 & 57 & 20 & 15 & 7.6 \\
\hline 84-2 & Ethanol & 230 & 1.42 & 16 & 61 & 18 & 11 & 9.8 \\
\hline $84-3$ & Ethanol & 230 & 2.06 & 13 & 64 & 16 & 8.4 & 11 \\
\hline $85-1$ & Ethanol & 250 & 0.79 & 40 & 51 & 20 & 18 & 8.7 \\
\hline $85-2$ & Ethanol & 250 & 1.42 & 30 & 54 & 18 & 16 & 8.8 \\
\hline
\end{tabular}




\begin{tabular}{|c|c|c|c|c|c|c|c|c|}
\hline $85-3$ & Ethanol & 250 & 2.06 & 26 & 55 & 19 & 12 & 9.3 \\
\hline $124-2$ & $\begin{array}{c}t \text {-butanol }+5 \mathrm{wt} \% \\
\text { acetaldehyde }\end{array}$ & 110 & 1.42 & 32 & 0.0 & 0.0 & 0.0 & 68 \\
\hline $124-3$ & $\begin{array}{c}t \text {-butanol }+5 \mathrm{wt} \% \\
\text { acetaldehyde }\end{array}$ & 120 & 1.42 & 55 & 0.0 & 0.0 & 0.0 & 75 \\
\hline $124-1$ & $\begin{array}{c}t \text {-butanol }+5 \mathrm{wt} \% \\
\text { acetaldehyde }\end{array}$ & 130 & 1.42 & 87 & 0.0 & 0.0 & 0.0 & 66 \\
\hline \multicolumn{9}{|c|}{$\mathrm{Ni}(\mathrm{D}): 8.0 \mathrm{wt} \% \mathrm{Ni} / 9.0 \mathrm{wt} \% \mathrm{La}_{2} \mathrm{O}_{3} / \gamma-\mathrm{Al}_{2} \mathrm{O}_{3}$} \\
\hline $99-1$ & Ethanol & 210 & 1.42 & 18 & 54 & 15 & 13 & 12 \\
\hline $99-2$ & Ethanol & 230 & 1.42 & 36 & 46 & 16 & 24 & 11 \\
\hline $99-3$ & Ethanol & 250 & 1.42 & 61 & 30 & 13 & 46 & 11 \\
\hline \multicolumn{9}{|c|}{$\mathrm{Ni}(\mathrm{E}): 1.0 \mathrm{wt} \% \mathrm{Ni} / 9.0 \mathrm{wt} \% \mathrm{La}_{2} \mathrm{O}_{3} / \gamma-\mathrm{Al}_{2} \mathrm{O}_{3}$} \\
\hline $111-1$ & Ethanol & 210 & 1.42 & 7.0 & 68 & 10 & 5.5 & 16 \\
\hline $109-1$ & Ethanol & 230 & 0.79 & 22 & 58 & 21 & 9.4 & 10 \\
\hline $109-2$ & Ethanol & 230 & 1.42 & 15 & 62 & 17 & 8.1 & 11 \\
\hline $109-3$ & Ethanol & 230 & 2.06 & 12 & 63 & 14 & 7.7 & 12 \\
\hline $110-1$ & Ethanol & 250 & 0.79 & 41 & 51 & 23 & 15 & 9.3 \\
\hline $110-2$ & Ethanol & 250 & 1.42 & 29 & 54 & 21 & 12 & 11 \\
\hline $110-3$ & Ethanol & 250 & 2.06 & 23 & 58 & 10 & 11 & 12 \\
\hline
\end{tabular}


Table S4. Selectivity to liquid byproducts for nickel catalysts

\begin{tabular}{|c|c|c|c|c|c|c|c|}
\hline \multirow{2}{*}{ Exp \# } & \multicolumn{7}{|c|}{ Selectivity (\%) } \\
\cline { 2 - 9 } & $\begin{array}{c}\text { Ethyl } \\
\text { Acetate }\end{array}$ & $\begin{array}{c}\text { Diethyl } \\
\text { Ether }\end{array}$ & Acetal & Acetaldehyde & Butyraldehyde & Crotonaldehyde & $\begin{array}{c}\text { Other } \\
\text { Liquids }\end{array}$ \\
\hline $116-2$ & 0.0 & 7.8 & 1.2 & 0.0 & 0.0 & 0.0 & 62 \\
\hline $116-1$ & 0.0 & 20 & 2.2 & 0.0 & 0.0 & 0.0 & 47 \\
\hline $116-3$ & 0.0 & 15 & 2.3 & 0.0 & 0.0 & 0.0 & 60 \\
\hline $28-4$ & 59 & 3.8 & 0.0 & 4.7 & 0.0 & 1.0 & 3.1 \\
\hline $28-1$ & 14 & 1.6 & 0.0 & 4.1 & 1.4 & 0.9 & 1.3 \\
\hline $48-2$ & 1.5 & 1.3 & 0.7 & 2.3 & 0.4 & 0.3 & 1.2 \\
\hline $29-3$ & 2.2 & 1.8 & 0.9 & 2.9 & 0.6 & 0.4 & 1.3 \\
\hline $29-2$ & 2.0 & 1.7 & 1.2 & 3.0 & 0.7 & 0.3 & 1.2 \\
\hline $28-2$ & 2.8 & 1.4 & 0.0 & 3.1 & 1.8 & 0.2 & 0.6 \\
\hline $29-1$ & 2.1 & 1.8 & 1.8 & 3.7 & 0.9 & 0.3 & 1.0 \\
\hline $48-1$ & 2.3 & 1.4 & 1.7 & 3.2 & 0.8 & 0.3 & 0.7 \\
\hline $54-1$ & 2.1 & 1.3 & 2.0 & -0.6 & 1.1 & 0.2 & 1.2 \\
\hline $56-1$ & 2.9 & 2.1 & 2.7 & 2.9 & 1.3 & 0.3 & 1.3 \\
\hline $58-1$ & 1.0 & 0.2 & 0.0 & 0.6 & 11 & 0.0 & 20 \\
\hline $65-1$ & 1.5 & 1.2 & 1.8 & 3.4 & 0.9 & 0.0 & 0.6 \\
\hline $48-3$ & 1.8 & 1.5 & 1.8 & 3.6 & 1.0 & 0.2 & 1.1 \\
\hline $28-3$ & 0.5 & 0.5 & 0.0 & 2.6 & 2.5 & 0.3 & 0.3 \\
\hline $66-1$ & 2.2 & 1.3 & 1.4 & 2.5 & 1.0 & 0.3 & 1.3 \\
\hline $83-1$ & 1.4 & 1.9 & 3.3 & 2.1 & 0.7 & 0.3 & 1.7 \\
\hline $83-2$ & 0.7 & 2.3 & 5.5 & 2.4 & 0.8 & 0.4 & 2.1 \\
\hline $83-3$ & 1.0 & 2.6 & 6.6 & 2.5 & 0.8 & 0.4 & 2.4 \\
\hline $84-1$ & 1.9 & 1.7 & 0.6 & 1.5 & 0.6 & 0.1 & 1.2 \\
\hline $84-2$ & 1.9 & 1.9 & 1.6 & 1.9 & 0.8 & 0.2 & 1.5 \\
\hline $84-3$ & 1.7 & 2.2 & 2.5 & 2.3 & 0.9 & 0.2 & 1.5 \\
\hline $85-1$ & 3.0 & 2.1 & 0.1 & 1.4 & 0.6 & 0.0 & 1.5 \\
\hline $85-2$ & 2.3 & 2.2 & 0.4 & 1.6 & 0.7 & 0.1 & 1.5 \\
\hline $85-3$ & 2.4 & 1.8 & 0.6 & 2.0 & 1.0 & 0.1 & 1.4 \\
\hline $124-2^{1}$ & 0.0 & 0.0 & 0.0 & - & 0.0 & 45 & 23 \\
\hline $124-31$ & 0.0 & 0.0 & 0.0 & - & 0.0 & 50 & 25 \\
\hline $124-1^{1}$ & 0.0 & 0.0 & 0.0 & - & 0.0 & 37 & 29 \\
\hline
\end{tabular}




\begin{tabular}{|c|c|c|c|c|c|c|c|}
\hline $99-1$ & 1.7 & 2.2 & 2.5 & 2.2 & 1.2 & 0.0 & 2.3 \\
\hline $99-2$ & 2.0 & 1.7 & 0.7 & 1.6 & 0.9 & 0.0 & 3.8 \\
\hline $99-3$ & 2.2 & 1.2 & 0.0 & 1.1 & 0.8 & 0.2 & 5.5 \\
\hline $111-1$ & 0.0 & 1.0 & 6.0 & 2.2 & 0.3 & 0.4 & 5.8 \\
\hline $109-1$ & 0.9 & 0.8 & 1.1 & 1.6 & 0.7 & 0.1 & 4.6 \\
\hline $109-2$ & 1.0 & 0.9 & 1.9 & 2.0 & 0.7 & 0.1 & 4.4 \\
\hline $109-3$ & 1.0 & 1.0 & 2.7 & 2.3 & 0.7 & 0.0 & 4.6 \\
\hline $110-1$ & 1.1 & 0.8 & 0.5 & 1.2 & 0.9 & 0.1 & 4.7 \\
\hline $110-2$ & 1.2 & 0.8 & 0.8 & 1.6 & 1.0 & 0.1 & 5.2 \\
\hline $110-3$ & 1.3 & 0.9 & 1.2 & 1.9 & 1.1 & 0.1 & 5.1 \\
\hline
\end{tabular}


Table S5. Selectivity to gas products and overall carbon recovery for nickel catalysts

\begin{tabular}{|c|c|c|c|c|c|c|}
\hline \multirow{2}{*}{ Exp \# } & \multicolumn{7}{|c|}{ Selectivity (\%) } \\
\cline { 2 - 7 } & Carbon Monoxide & Carbon Dioxide & Methane & Ethane & Propane & $\begin{array}{c}\text { Overall carbon } \\
\text { recovery }\end{array}$ \\
\hline $28-4$ & 0.1 & 1.3 & 18 & 0.5 & 0.2 & 123 \\
\hline $28-1$ & 0.1 & 0.6 & 9.2 & 0.5 & 0.4 & 100 \\
\hline $48-2$ & 0.2 & 5.0 & 16 & 2.1 & 3.7 & 95 \\
\hline $29-3$ & 0.6 & 3.6 & 14 & 0.9 & 2.4 & 105 \\
\hline $29-2$ & 0.4 & 2.6 & 12 & 0.8 & 1.7 & 98 \\
\hline $28-2$ & 0.3 & 2.0 & 12 & 0.7 & 1.3 & 99 \\
\hline $29-1$ & 1.0 & 2.2 & 11 & 0.7 & 1.5 & 104 \\
\hline $48-1$ & 0.2 & 2.0 & 11 & 1.2 & 1.9 & 96 \\
\hline $54-1$ & 0.1 & 2.0 & 9.0 & 1.2 & 2.6 & 96 \\
\hline $56-1$ & 0.1 & 1.8 & 11 & 1.6 & 2.4 & 98 \\
\hline $58-1$ & 0.1 & 1.2 & 2.4 & 0.7 & 15 & 85 \\
\hline $65-1$ & 0.3 & 3.7 & 16 & 1.5 & 3.0 & 97 \\
\hline $48-3$ & 0.1 & 1.7 & 11 & 1.1 & 1.7 & 95 \\
\hline $28-3$ & 0.4 & 5.3 & 18 & 1.2 & 3.6 & 96 \\
\hline $66-1$ & 0.2 & 2.3 & 11 & 1.4 & 3.3 & 96 \\
\hline $83-1$ & 0.0 & 0.7 & 6.0 & 1.9 & 1.3 & 96 \\
\hline $83-2$ & 0.1 & 0.5 & 6.4 & 1.5 & 0.2 & 96 \\
\hline $83-3$ & 0.0 & 0.4 & 6.2 & 1.3 & 0.0 & 94 \\
\hline $84-1$ & 0.1 & 1.9 & 6.2 & 2.3 & 2.0 & 97 \\
\hline $84-2$ & 0.1 & 1.1 & 6.2 & 2.1 & 1.7 & 100 \\
\hline $84-3$ & 0.1 & 0.6 & 6.5 & 1.6 & 0.0 & 100 \\
\hline $85-1$ & 0.2 & 1.9 & 10 & 3.0 & 3.1 & 97 \\
\hline $85-2$ & 0.7 & 2.0 & 7.8 & 2.1 & 3.1 & 96 \\
\hline $85-3$ & 0.3 & 0.9 & 8.2 & 1.8 & 1.0 & 95 \\
\hline $124-2$ & 0.0 & 0.0 & 0.0 & 0.0 & 0.0 & 73 \\
\hline $124-3$ & 0.0 & 0.0 & 0.0 & 0.0 & 0.0 & 77 \\
\hline $124-1$ & 0.0 & 0.0 & 0.0 & 0.0 & 0.0 & 68 \\
\hline $99-1$ & 0.4 & 1.1 & 7.9 & 1.4 & 2.1 & 94 \\
\hline $99-2$ & 0.6 & 2.9 & 11 & 3.4 & 5.9 & 96 \\
\hline $99-3$ & 1.5 & 6.6 & 20 & 3.8 & 15 & 100 \\
\hline & & & & & & \\
\hline
\end{tabular}




\begin{tabular}{|l|l|l|l|l|l|l|}
\hline $111-1$ & 0.1 & 0.2 & 4.6 & 0.2 & 0.2 & 99 \\
\hline $109-1$ & 0.2 & 1.3 & 6.0 & 0.7 & 1.3 & 98 \\
\hline $109-2$ & 0.2 & 0.8 & 5.8 & 0.5 & 0.8 & 98 \\
\hline $109-3$ & 0.2 & 0.7 & 5.7 & 0.4 & 0.7 & 97 \\
\hline $110-1$ & 0.2 & 2.6 & 8.0 & 0.8 & 3.1 & 98 \\
\hline $110-2$ & 0.2 & 1.9 & 7.3 & 0.7 & 2.3 & 98 \\
\hline $110-3$ & 0.1 & 5.2 & 4.2 & 0.7 & 1.0 & 91 \\
\hline
\end{tabular}


Table S6. Nickel and bimetallic catalysts BET surface area.

\begin{tabular}{|c|c|}
\hline Catalyst & $\begin{array}{c}\text { BET Surface Area }( \pm \mathbf{5}) \\
\left(\mathbf{m}^{2} / \mathbf{g}\right)\end{array}$ \\
\hline$\gamma-\mathrm{Al}_{2} \mathrm{O}_{3}$ & 146 \\
\hline $9.0 \mathrm{wt} \% \mathrm{La}_{2} \mathrm{O}_{3} / \gamma-\mathrm{Al}_{2} \mathrm{O}_{3}$ & 143 \\
\hline $\mathrm{Ni}(\mathrm{A})$ & 138 \\
\hline $\mathrm{Ni}(\mathrm{B})$ & 133 \\
\hline $\mathrm{Ni}(\mathrm{C})$ & 126 \\
\hline $\mathrm{Ni}(\mathrm{D})$ & 129 \\
\hline $\mathrm{Ni}(\mathrm{E})$ & 140 \\
\hline $\begin{array}{c}11.5 \mathrm{wt} \%(\mathrm{Ni} / \mathrm{Cu} 2 / 1) / 8.7 \\
\mathrm{wt} \% \mathrm{La} \mathrm{O}_{3} / \gamma-\mathrm{Al}_{2} \mathrm{O}_{3}\end{array}$ & 118 \\
\hline $\begin{array}{c}8.4 \mathrm{wt} \%(\mathrm{Ni} / \mathrm{Cu} 20 / 1) / 9.0 \\
\mathrm{wt} \% \mathrm{La}_{2} \mathrm{O}_{3} / \gamma-\mathrm{Al}_{2} \mathrm{O}_{3}\end{array}$ & 121 \\
\hline $\begin{array}{c}11.5 \mathrm{wt} \%(\mathrm{Ni} / \mathrm{Co} 2 / 1) / 8.7 \\
\mathrm{wt} \% \mathrm{La}_{2} \mathrm{O}_{3} / \gamma-\mathrm{Al}_{2} \mathrm{O}_{3}\end{array}$ & 119 \\
\hline $\begin{array}{c}9.7 \mathrm{wt} \%(\mathrm{Ni} / \mathrm{Pd} 4 / 1) / 8.8 \\
\mathrm{wt} \% \mathrm{La}_{2} \mathrm{O}_{3} / \gamma-\mathrm{Al}_{2} \mathrm{O}_{3}\end{array}$ & 123 \\
\hline $\begin{array}{c}9.7 \mathrm{wt} \%(\mathrm{Ni} / \mathrm{Pt} 4 / 1) / 8.8 \mathrm{wt} \% \\
\mathrm{~L}_{2} \mathrm{O}_{3} / \gamma-\mathrm{Al}_{2} \mathrm{O}_{3}\end{array}$ & 124 \\
\hline $\begin{array}{c}11.5 \mathrm{wt} \%(\mathrm{Ni} / \mathrm{Fe} 2 / 1) / 8.7 \\
\mathrm{wt} \% \mathrm{La}_{2} \mathrm{O}_{3} / \gamma-\mathrm{Al}_{2} \mathrm{O}_{3}\end{array}$ & 117 \\
\hline $\begin{array}{c}11.5 \mathrm{wt} \%\left(\mathrm{Ni} / \mathrm{Mo}_{2} / 1\right) / 8.7 \\
\mathrm{wt} \% \mathrm{La}_{2} \mathrm{O}_{3} / \gamma-\mathrm{Al}_{2} \mathrm{O}_{3}\end{array}$ & 123 \\
\hline
\end{tabular}


Table S7. Experimental conditions and summary of results for bimetallic catalysts. Bimetallic catalysts prepared by sequential impregnation unless otherwise noted.

\begin{tabular}{|c|c|c|c|c|c|c|c|c|}
\hline \multirow[b]{2}{*}{ Exp \# } & \multirow{2}{*}{$\begin{array}{c}\mathrm{T} \\
\left({ }^{\circ} \mathrm{C}\right)\end{array}$} & \multirow{2}{*}{$\begin{array}{c}\text { WHSV } \\
\left(\mathrm{h}^{-1}\right)\end{array}$} & \multirow{2}{*}{$\begin{array}{c}\text { Ethanol } \\
\text { Conv. } \\
(\%)\end{array}$} & \multicolumn{5}{|c|}{ Selectivity (\%) } \\
\hline & & & & $\begin{array}{c}n- \\
\mathrm{BuOH} \\
\end{array}$ & $\mathrm{C}_{6+} \mathrm{OH}$ & Gas & $\begin{array}{l}\text { Ethyl } \\
\text { acetate }\end{array}$ & $\begin{array}{l}\text { Other liquid } \\
\text { byproducts }\end{array}$ \\
\hline \multicolumn{9}{|c|}{$11.5 \mathrm{wt} \%(\mathrm{Ni} / \mathrm{Cu} 2 / 1) / 8.7 \mathrm{wt} \% \mathrm{La}_{2} \mathrm{O}_{3} / \gamma-\mathrm{Al}_{2} \mathrm{O}_{3}$} \\
\hline $86-1$ & 230 & 0.79 & 22 & 64 & 7.7 & 15 & 1.4 & 10 \\
\hline $86-2$ & 230 & 1.42 & 17 & 66 & 7.0 & 13 & 1.4 & 10 \\
\hline $86-3$ & 230 & 2.06 & 14 & 67 & 6.8 & 12 & 1.4 & 11 \\
\hline $87-1$ & 250 & 0.79 & 39 & 54 & 11 & 23 & 2.1 & 7.4 \\
\hline $87-2$ & 250 & 1.42 & 29 & 62 & 10 & 16 & 2.4 & 7.1 \\
\hline $87-3$ & 250 & 2.06 & 25 & 62 & 10 & 14 & 2.7 & 7.4 \\
\hline \multicolumn{9}{|c|}{$11.5 \mathrm{wt} \%(\mathrm{Ni} / \mathrm{Cu} 2 / 1) / 8.7 \mathrm{wt} \% \mathrm{La}_{2} \mathrm{O}_{3} / \gamma-\mathrm{Al}_{2} \mathrm{O}_{3}(\mathrm{Co}-$ Impregnated $)$} \\
\hline $74-1$ & 210 & 1.42 & 7.3 & 67 & 3.1 & 10 & 1.1 & 16 \\
\hline $76-3$ & 230 & 1.42 & 17 & 71 & 6.4 & 11 & 2.3 & 8.7 \\
\hline $76-2$ & 250 & 1.42 & 30 & 62 & 8.6 & 15 & 2.3 & 8.2 \\
\hline $76-3$ & 250 & 3.32 & 22 & 66 & 8.0 & 10 & 2.6 & 8.3 \\
\hline \multicolumn{9}{|c|}{$8.4 \mathrm{wt} \%(\mathrm{Ni} / \mathrm{Cu} 20 / 1) / 9.0 \mathrm{wt} \% \mathrm{La}_{2} \mathrm{O}_{3} / \gamma-\mathrm{Al}_{2} \mathrm{O}_{3}$ (Co-Impregnated) } \\
\hline $79-3$ & 210 & 0.79 & 11 & 68 & 9.1 & 8.7 & 1.1 & 9.5 \\
\hline $78-2$ & 210 & 1.42 & 8.8 & 68 & 7.6 & 8.2 & 1.7 & 11 \\
\hline $80-1$ & 210 & 2.06 & 7.6 & 65 & 7.0 & 8.1 & 1.6 & 12 \\
\hline $79-2$ & 230 & 0.79 & 26 & 60 & 12 & 17 & 2.4 & 5.8 \\
\hline $78-1$ & 230 & 1.42 & 18 & 65 & 11 & 12 & 2.7 & 7.0 \\
\hline $80-2$ & 230 & 2.06 & 15 & 66 & 12 & 9.2 & 1.8 & 8.0 \\
\hline $79-1$ & 250 & 0.79 & 44 & 52 & 15 & 21 & 3.4 & 4.8 \\
\hline $80-4$ & 250 & 1.42 & 35 & 59 & 15 & 17 & 2.8 & 5.5 \\
\hline $80-3$ & 250 & 2.06 & 29 & 58 & 14 & 15 & 2.9 & 5.9 \\
\hline \multicolumn{9}{|c|}{$8.0 \mathrm{wt} \% \mathrm{Cu} / 9.0 \mathrm{wt} \% \mathrm{La}_{2} \mathrm{O}_{3} / \gamma-\mathrm{Al}_{2} \mathrm{O}_{3}$} \\
\hline $97-1$ & 210 & 1.42 & 6.6 & 5.2 & 0.0 & 4.1 & 67 & 16 \\
\hline $95-1$ & 230 & 0.79 & 13 & 5.2 & 0.0 & 5.1 & 62 & 19 \\
\hline $95-2$ & 230 & 1.42 & 10 & 5.6 & 0.0 & 5.3 & 62 & 21 \\
\hline $95-3$ & 230 & 2.06 & 9.3 & 5.7 & 0.0 & 5.8 & 62 & 20 \\
\hline $96-1$ & 250 & 0.79 & 26 & 6.7 & 0.0 & 9.9 & 52 & 24 \\
\hline $96-2$ & 250 & 1.42 & 20 & 7.3 & 0.0 & 5.8 & 56 & 24 \\
\hline $96-3$ & 250 & 2.06 & 19 & 6.7 & 0.0 & 16 & 52 & 21 \\
\hline
\end{tabular}




\begin{tabular}{|c|c|c|c|c|c|c|c|c|}
\hline \multicolumn{9}{|c|}{$11.5 \mathrm{wt} \%(\mathrm{Ni} / \mathrm{Co} 2 / 1) / 8.7 \mathrm{wt} \% \mathrm{La}_{2} \mathrm{O}_{3} / \gamma-\mathrm{Al}_{2} \mathrm{O}_{3}$} \\
\hline $105-1$ & 210 & 1.42 & 12 & 57 & 15.3 & 9.3 & 0.0 & 11 \\
\hline $103-1$ & 230 & 0.79 & 46 & 41 & 24.6 & 19 & 1.4 & 10 \\
\hline $103-2$ & 230 & 1.42 & 33 & 43 & 22.9 & 16 & 1.4 & 10 \\
\hline $103-3$ & 230 & 2.06 & 27 & 46 & 21.9 & 14 & 1.3 & 10 \\
\hline $104-1$ & 250 & 0.79 & 60 & 22 & 11.9 & 46 & 1.3 & 11 \\
\hline $104-2$ & 250 & 1.42 & 51 & 34 & 17.8 & 29 & 1.5 & 11 \\
\hline $104-3$ & 250 & 2.06 & 43 & 42 & 20.1 & 16 & 1.7 & 11 \\
\hline \multicolumn{9}{|c|}{$8.0 \mathrm{wt} \% \mathrm{Co} / 9.0 \mathrm{wt} \% \mathrm{La}_{2} \mathrm{O}_{3} / \gamma-\mathrm{Al}_{2} \mathrm{O}_{3}$} \\
\hline $115-2$ & 210 & 1.42 & 13 & 7.5 & 0.0 & 21 & 52 & 15 \\
\hline $114-1$ & 230 & 0.79 & 38 & 0.7 & 0.0 & 25 & 63 & 4.7 \\
\hline $115-3$ & 230 & 1.42 & 24 & 6.5 & 0.1 & 22 & 53 & 14 \\
\hline $115-1$ & 250 & 1.42 & 49 & 4.3 & 0.1 & 24 & 55 & 10 \\
\hline \multicolumn{9}{|c|}{$9.7 \mathrm{wt} \%(\mathrm{Ni} / \mathrm{Pd} 4 / 1) / 8.8 \mathrm{wt} \% \mathrm{La}_{2} \mathrm{O}_{3} / \gamma-\mathrm{Al}_{2} \mathrm{O}_{3}$} \\
\hline $107-1$ & 210 & 1.42 & 9.9 & 54 & 8.9 & 5.9 & 0.9 & 25 \\
\hline $106-1$ & 230 & 0.79 & 28 & 51 & 15.7 & 14 & 1.3 & 14 \\
\hline $106-2$ & 230 & 1.42 & 20 & 53 & 13.8 & 12 & 1.1 & 15 \\
\hline $106-3$ & 230 & 2.06 & 17 & 53 & 12.1 & 12 & 1.1 & 18 \\
\hline $108-1$ & 250 & 0.79 & 47 & 41 & 14.9 & 24 & 1.8 & 14 \\
\hline $108-2$ & 250 & 1.42 & 34 & 47 & 14.2 & 13 & 1.7 & 16 \\
\hline $108-3$ & 250 & 2.06 & 28 & 49 & 13.3 & 12 & 1.4 & 18 \\
\hline \multicolumn{9}{|c|}{$9.7 \mathrm{wt} \%(\mathrm{Ni} / \mathrm{Pt} 4 / 1) / 8.8 \mathrm{wt} \% \mathrm{La}_{2} \mathrm{O}_{3} / \gamma-\mathrm{Al}_{2} \mathrm{O}_{3}$} \\
\hline 91-1 & 210 & 1.42 & 9.2 & 56 & 4.9 & 20 & 0.0 & 11 \\
\hline 89-1 & 230 & 0.79 & 28 & 44 & 7.3 & 36 & 1.4 & 7.4 \\
\hline $89-2$ & 230 & 1.42 & 21 & 49 & 7.6 & 31 & 1.5 & 7.7 \\
\hline 89-3 & 230 & 2.06 & 17 & 51 & 7.3 & 29 & 1.4 & 8.5 \\
\hline $90-1$ & 250 & 0.79 & 59 & 20 & 4.4 & 66 & 0.8 & 3.9 \\
\hline $90-2$ & 250 & 1.42 & 41 & 33 & 6.8 & 48 & 1.4 & 5.1 \\
\hline $90-3$ & 250 & 2.06 & 35 & 35 & 7.9 & 46 & 1.4 & 5.0 \\
\hline \multicolumn{9}{|c|}{$11.5 \mathrm{wt} \%(\mathrm{Ni} / \mathrm{Fe} 2 / 1) / 8.7 \mathrm{wt} \% \mathrm{La}_{2} \mathrm{O}_{3} / \gamma-\mathrm{Al}_{2} \mathrm{O}_{3}$} \\
\hline 94-1 & 210 & 1.42 & 8.9 & 46 & 1.8 & 15 & 7.6 & 22 \\
\hline $92-1$ & 230 & 0.79 & 30 & 43 & 5.6 & 27 & 7.3 & 14 \\
\hline $92-2$ & 230 & 1.42 & 22 & 44 & 5.0 & 24 & 7.6 & 16 \\
\hline $92-3$ & 230 & 2.06 & 18 & 44 & 4.3 & 22 & 6.4 & 19 \\
\hline
\end{tabular}




\begin{tabular}{|c|c|c|c|c|c|c|c|c|}
\hline $93-1$ & 250 & 0.79 & 44 & 37 & 8.7 & 31 & 6.5 & 13 \\
\hline $93-2$ & 250 & 1.42 & 32 & 44 & 9.6 & 23 & 5.1 & 13 \\
\hline $93-3$ & 250 & 2.06 & 26 & 47 & 9.3 & 20 & 5.3 & 14 \\
\hline \multicolumn{7}{|c|}{$11.5 \mathrm{wt} \%$ (Ni/Mo $2 / 1) / 8.7$} & $\mathrm{wt} \% \mathrm{La}_{2} \mathrm{O}_{3} / \gamma-\mathrm{Al}_{2} \mathrm{O}_{3}$ \\
\hline $102-1$ & 210 & 1.42 & 5.2 & 51 & 2.1 & 5.9 & 7.3 & 21 \\
\hline $100-1$ & 230 & 0.79 & 9.7 & 56 & 2.4 & 11 & 11 & 13 \\
\hline $100-2$ & 230 & 1.42 & 9.3 & 51 & 2.4 & 10 & 14 & 13 \\
\hline $100-3$ & 230 & 2.06 & 8.1 & 52 & 2.6 & 11 & 11 & 15 \\
\hline $101-1$ & 250 & 0.79 & 17 & 55 & 5.0 & 1.8 & 13 & 20 \\
\hline $101-2$ & 250 & 1.42 & 15 & 53 & 4.8 & 9.9 & 7.5 & 20 \\
\hline $101-3$ & 250 & 2.06 & 13 & 55 & 4.9 & 7.1 & 10 & 17 \\
\hline
\end{tabular}


Table S8. Selectivity to liquid byproducts for bimetallic catalysts

\begin{tabular}{|c|c|c|c|c|c|c|c|}
\hline \multirow[b]{2}{*}{ Exp \# } & \multicolumn{7}{|c|}{ Selectivity (\%) } \\
\hline & $\begin{array}{c}\text { Ethyl } \\
\text { Acetate }\end{array}$ & $\begin{array}{l}\text { Diethyl } \\
\text { Ether }\end{array}$ & Acetal & Acetaldehyde & Butyraldehyde & Crotonaldehyde & $\begin{array}{l}\text { Other } \\
\text { Liquids }\end{array}$ \\
\hline 86-1 & 1.4 & 6.4 & 1.5 & 1.3 & 0.0 & 0.0 & 1.0 \\
\hline $86-2$ & 1.4 & 5.4 & 2.0 & 1.7 & 0.0 & 0.0 & 1.1 \\
\hline $86-3$ & 1.4 & 5.1 & 2.5 & 2.0 & 0.0 & 0.0 & 1.1 \\
\hline $87-1$ & 2.1 & 4.5 & 0.0 & 1.2 & 0.4 & 0.0 & 1.3 \\
\hline $87-2$ & 2.4 & 4.6 & 0.0 & 1.4 & 0.0 & 0.0 & 1.1 \\
\hline $87-3$ & 2.7 & 4.5 & 0.0 & 1.7 & 0.2 & 0.0 & 1.0 \\
\hline 74-1 & 1.1 & 7.5 & 5.3 & 1.2 & 0.0 & 0.0 & 1.9 \\
\hline $76-3$ & 2.3 & 5.0 & 1.4 & 1.2 & 0.0 & 0.0 & 1.1 \\
\hline $76-2$ & 2.3 & 4.5 & 0.7 & 1.0 & 0.3 & 0.0 & 1.7 \\
\hline $76-3$ & 2.6 & 3.9 & 0.9 & 1.6 & 0.4 & 0.0 & 1.5 \\
\hline $79-3$ & 1.1 & 2.8 & 3.2 & 1.3 & 0.2 & 0.3 & 1.7 \\
\hline $78-2$ & 1.7 & 2.7 & 4.2 & 1.6 & 0.2 & 0.4 & 1.7 \\
\hline $80-1$ & 1.6 & 2.6 & 5.3 & 1.7 & 0.2 & 0.4 & 1.9 \\
\hline $79-2$ & 2.4 & 2.3 & 0.8 & 0.9 & 0.4 & 0.1 & 1.3 \\
\hline $78-1$ & 2.7 & 2.2 & 1.4 & 1.4 & 0.4 & 0.2 & 1.4 \\
\hline $80-2$ & 1.8 & 2.3 & 1.9 & 1.6 & 0.5 & 0.3 & 1.4 \\
\hline $79-1$ & 3.4 & 1.9 & 0.1 & 0.9 & 0.5 & 0.0 & 1.4 \\
\hline $80-4$ & 2.8 & 2.0 & 0.4 & 1.2 & 0.6 & 0.1 & 1.2 \\
\hline $80-3$ & 2.9 & 2.0 & 0.5 & 1.4 & 0.7 & 0.1 & 1.2 \\
\hline $97-1$ & 67 & 0.6 & 0.0 & 0.0 & 0.0 & 0.0 & 15 \\
\hline $95-1$ & 62 & 1.8 & 0.0 & 0.0 & 0.0 & 0.0 & 18 \\
\hline $95-2$ & 62 & 1.3 & 0.0 & 0.0 & 0.0 & 0.0 & 20 \\
\hline $95-3$ & 62 & 1.1 & 0.0 & 0.0 & 0.0 & 0.0 & 19 \\
\hline 96-1 & 52 & 2.1 & 0.0 & 0.6 & 0.0 & 0.0 & 21 \\
\hline $96-2$ & 56 & 1.6 & 0.0 & 0.7 & 0.0 & 0.0 & 21 \\
\hline 96-3 & 52 & 1.1 & 0.0 & 0.5 & 0.0 & 0.0 & 19 \\
\hline $105-1$ & 0.0 & 0.3 & 4.4 & 0.0 & 0.0 & 0.0 & 6.2 \\
\hline 103-1 & 1.4 & 0.4 & 0.4 & 0.9 & 1.1 & 0.0 & 7.3 \\
\hline 103-2 & 1.4 & 0.4 & 0.8 & 0.8 & 1.6 & 0.0 & 6.6 \\
\hline $103-3$ & 1.3 & 0.5 & 1.1 & 0.7 & 1.8 & 0.0 & 6.3 \\
\hline 104-1 & 1.3 & 0.4 & 0.3 & 2.4 & 0.9 & 0.0 & 7.3 \\
\hline $104-2$ & 1.5 & 0.4 & 0.3 & 1.4 & 1.3 & 0.0 & 7.3 \\
\hline
\end{tabular}




\begin{tabular}{|c|c|c|c|c|c|c|c|}
\hline $104-3$ & 1.7 & 0.5 & 0.4 & 1.0 & 1.7 & 0.0 & 6.9 \\
\hline $115-2$ & 52 & 1.8 & 0.0 & 0.6 & 0.0 & 0.0 & 13 \\
\hline 114-1 & 63 & 1.7 & 0.0 & 0.3 & 0.0 & 0.0 & 2.7 \\
\hline $115-3$ & 53 & 2.4 & 0.0 & 0.4 & 0.0 & 0.0 & 11 \\
\hline $115-1$ & 55 & 1.2 & 0.0 & 0.5 & 0.0 & 0.0 & 8.4 \\
\hline $107-1$ & 0.9 & 12 & 3.7 & 1.9 & 0.6 & 0.1 & 6.3 \\
\hline $106-1$ & 1.3 & 5.8 & 0.7 & 1.7 & 0.7 & 0.1 & 4.9 \\
\hline $106-2$ & 1.1 & 6.6 & 1.3 & 2.0 & 0.8 & 0.1 & 4.4 \\
\hline $106-3$ & 1.1 & 8.2 & 1.8 & 2.3 & 0.9 & 0.1 & 4.3 \\
\hline $108-1$ & 1.8 & 4.9 & 0.0 & 1.0 & 0.7 & 0.1 & 7.3 \\
\hline $108-2$ & 1.7 & 6.5 & 0.3 & 1.4 & 0.7 & 0.1 & 7.3 \\
\hline $108-3$ & 1.4 & 7.4 & 0.7 & 1.6 & 0.8 & 0.1 & 7.2 \\
\hline $91-1$ & 0.0 & 4.2 & 4.6 & 1.8 & 0.0 & 0.0 & 0.9 \\
\hline $89-1$ & 1.4 & 4.7 & 0.7 & 1.1 & 0.0 & 0.0 & 0.9 \\
\hline $89-2$ & 1.5 & 4.5 & 1.0 & 1.3 & 0.0 & 0.0 & 0.9 \\
\hline $89-3$ & 1.4 & 4.5 & 1.4 & 1.6 & 0.0 & 0.0 & 1.0 \\
\hline $90-1$ & 0.8 & 2.3 & 0.2 & 0.4 & 0.0 & 0.0 & 1.0 \\
\hline $90-2$ & 1.4 & 3.0 & 0.4 & 0.8 & 0.0 & 0.0 & 0.9 \\
\hline $90-3$ & 1.4 & 2.5 & 0.5 & 1.1 & 0.0 & 0.0 & 0.9 \\
\hline $94-1$ & 7.6 & 7.9 & 9.3 & 2.8 & 0.0 & 0.0 & 2.3 \\
\hline $92-1$ & 7.3 & 7.1 & 0.7 & 2.0 & 0.6 & 0.6 & 2.9 \\
\hline $92-2$ & 7.6 & 8.0 & 1.0 & 2.7 & 0.9 & 0.6 & 2.5 \\
\hline $92-3$ & 6.4 & 9.3 & 3.1 & 3.3 & 1.0 & 0.0 & 2.2 \\
\hline $93-1$ & 6.5 & 4.8 & 0.0 & 1.6 & 0.8 & 0.7 & 4.7 \\
\hline $93-2$ & 5.1 & 5.2 & 0.5 & 2.1 & 1.1 & 0.7 & 3.8 \\
\hline $93-3$ & 5.3 & 5.7 & 0.5 & 2.5 & 1.2 & 0.7 & 3.1 \\
\hline $102-1$ & 7.3 & 0.7 & 9.6 & 1.8 & 0.0 & 0.0 & 9.4 \\
\hline $100-1$ & 11 & 2.2 & 1.3 & 1.4 & 0.0 & 0.0 & 8.1 \\
\hline $100-2$ & 14 & 1.3 & 4.3 & 1.5 & 0.0 & 0.0 & 6.2 \\
\hline $100-3$ & 11 & 0.9 & 6.1 & 1.8 & 0.0 & 0.0 & 6.4 \\
\hline $101-1$ & 13 & 2.2 & 0.0 & 1.2 & 0.0 & 0.0 & 17 \\
\hline $101-2$ & 7.5 & 1.6 & 0.6 & 1.5 & 0.0 & 0.0 & 16 \\
\hline $101-3$ & 10 & 1.5 & 0.2 & 1.9 & 0.0 & 0.0 & 14 \\
\hline
\end{tabular}


Table S9. Selectivities to gas products and overall carbon recovery for bimetallic catalysts

\begin{tabular}{|c|c|c|c|c|c|c|}
\hline \multirow{2}{*}{ Exp \# } & \multicolumn{7}{|c|}{ Selectivity (\%) } \\
\cline { 2 - 7 } & Carbon Monoxide & Carbon Dioxide & Methane & Ethane & Propane & $\begin{array}{c}\text { Overall carbon } \\
\text { recovery (\%) }\end{array}$ \\
\hline $86-1$ & 0.1 & 2.5 & 8.2 & 2.4 & 1.4 & 98 \\
\hline $86-2$ & 0.2 & 2.0 & 7.1 & 2.4 & 1.0 & 97 \\
\hline $86-3$ & 0.2 & 1.8 & 7.0 & 1.6 & 0.9 & 97 \\
\hline $87-1$ & 0.2 & 5.1 & 11 & 2.6 & 3.5 & 97 \\
\hline $87-2$ & 0.2 & 3.2 & 7.6 & 2.8 & 2.0 & 97 \\
\hline $87-3$ & 0.2 & 2.9 & 7.2 & 2.0 & 1.8 & 97 \\
\hline $74-1$ & 0.2 & 1.3 & 7.8 & 0.6 & 0.1 & 97 \\
\hline $76-3$ & 0.1 & 2.9 & 7.2 & 0.8 & 0.6 & 100 \\
\hline $76-2$ & 0.2 & 4.2 & 7.8 & 0.9 & 1.8 & 96 \\
\hline $76-3$ & 0.2 & 2.6 & 5.1 & 1.2 & 1.1 & 95 \\
\hline $79-3$ & 0.2 & 0.9 & 6.3 & 1.1 & 0.2 & 96 \\
\hline $78-2$ & 0.2 & 0.7 & 6.5 & 0.5 & 0.2 & 96 \\
\hline $80-1$ & 0.4 & 0.8 & 5.9 & 0.6 & 0.2 & 94 \\
\hline $79-2$ & 0.2 & 2.4 & 10 & 1.4 & 2.1 & 97 \\
\hline $78-1$ & 0.2 & 1.2 & 8.3 & 0.9 & 1.3 & 98 \\
\hline $80-2$ & 0.2 & 1.1 & 6.9 & 0.8 & 0.1 & 97 \\
\hline $79-1$ & 0.4 & 4.4 & 12 & 1.5 & 1.1 & 95 \\
\hline $80-4$ & 0.4 & 2.9 & 9.9 & 1.5 & 2.8 & 100 \\
\hline $80-3$ & 0.3 & 2.5 & 9.3 & 1.3 & 1.9 & 97 \\
\hline $97-1$ & 0.9 & 0.5 & 1.6 & 1.0 & 0.0 & 92 \\
\hline $95-1$ & 1.2 & 0.3 & 1.8 & 1.7 & 0.0 & 92 \\
\hline $95-2$ & 1.0 & 0.2 & 2.6 & 1.5 & 0.0 & 94 \\
\hline $95-3$ & 0.9 & 0.6 & 2.8 & 1.5 & 0.0 & 94 \\
\hline $96-1$ & 1.5 & 2.2 & 3.2 & 2.9 & 0.0 & 92 \\
\hline $96-2$ & 1.4 & 1.3 & 1.2 & 1.8 & 0.0 & 93 \\
\hline $96-3$ & 1.2 & 2.1 & 8.5 & 2.7 & 1.3 & 95 \\
\hline $105-1$ & 0.2 & 0.6 & 6.1 & 1.1 & 1.3 & 92 \\
\hline $103-1$ & 0.4 & 2.6 & 8.0 & 1.1 & 6.5 & 96 \\
\hline $103-2$ & 0.4 & 1.8 & 7.4 & 1.1 & 5.0 & 93 \\
\hline $103-3$ & 0.4 & 1.2 & 7.0 & 1.3 & 4.0 & 94 \\
\hline $104-1$ & 0.9 & 7.7 & 19 & 2.9 & 15.9 & 93 \\
\hline $104-2$ & 0.6 & 4.2 & 12 & 2.6 & 9.6 & 93 \\
\hline & & & & & & \\
\hline
\end{tabular}




\begin{tabular}{|c|c|c|c|c|c|c|}
\hline $104-3$ & 0.2 & 2.6 & 6.3 & 0.9 & 6.1 & 90 \\
\hline $115-2$ & 1.6 & 0.0 & 17 & 0.0 & 2.0 & 96 \\
\hline $114-1$ & 2.2 & 0.0 & 21 & 0.0 & 1.9 & 94 \\
\hline $115-3$ & 1.8 & 0.0 & 18 & 0.0 & 2.2 & 95 \\
\hline $115-1$ & 2.1 & 0.2 & 18 & 0.0 & 3.0 & 94 \\
\hline $107-1$ & 0.1 & 0.3 & 3.5 & 1.5 & 0.5 & 95 \\
\hline $106-1$ & 0.3 & 1.7 & 6.1 & 3.0 & 2.9 & 96 \\
\hline $106-2$ & 0.2 & 1.2 & 5.4 & 3.1 & 2.2 & 96 \\
\hline $106-3$ & 0.2 & 1.0 & 5.6 & 3.2 & 1.7 & 96 \\
\hline $108-1$ & 0.4 & 3.4 & 8.6 & 5.5 & 6.2 & 96 \\
\hline $108-2$ & 0.2 & 1.6 & 4.7 & 3.9 & 2.9 & 93 \\
\hline $108-3$ & 0.2 & 1.3 & 4.6 & 3.5 & 2.5 & 94 \\
\hline $91-1$ & 0.2 & 3.7 & 12 & 2.4 & 1.3 & 92 \\
\hline 89-1 & 0.5 & 9.0 & 19 & 3.8 & 4.3 & 96 \\
\hline $89-2$ & 0.4 & 7.3 & 16 & 4.0 & 3.7 & 97 \\
\hline $89-3$ & 0.4 & 6.2 & 15 & 4.2 & 3.2 & 97 \\
\hline $90-1$ & 0.3 & 18 & 30 & 7.0 & 11 & 95 \\
\hline $90-2$ & 0.5 & 15 & 24 & 5.3 & 3.5 & 94 \\
\hline $90-3$ & 0.5 & 12 & 20 & 6.4 & 6.8 & 96 \\
\hline 94-1 & 0.1 & 0.4 & 11 & 2.9 & 1.0 & 93 \\
\hline $92-1$ & 0.2 & 3.7 & 15 & 4.4 & 4.1 & 97 \\
\hline $92-2$ & 0.2 & 2.7 & 15 & 2.9 & 3.4 & 96 \\
\hline $92-3$ & 0.2 & 2.0 & 14 & 2.7 & 2.7 & 96 \\
\hline $93-1$ & 0.1 & 4.8 & 15 & 5.4 & 6.3 & 97 \\
\hline $93-2$ & 0.2 & 3.0 & 13 & 3.0 & 4.2 & 96 \\
\hline $93-3$ & 0.2 & 2.3 & 11 & 3.0 & 3.5 & 96 \\
\hline $102-1$ & 0.0 & 0.1 & 1.4 & 4.3 & 0.1 & 88 \\
\hline $100-1$ & 0.6 & 0.2 & 1.4 & 9.0 & 0.0 & 94 \\
\hline $100-2$ & 0.6 & 0.4 & 2.8 & 6.4 & 0.2 & 92 \\
\hline $100-3$ & 0.6 & 0.6 & 4.4 & 4.7 & 0.7 & 92 \\
\hline $101-1$ & 0.2 & 0.1 & 0.3 & 1.2 & 0.0 & 95 \\
\hline $101-2$ & 0.6 & 1.0 & 2.8 & 4.6 & 1.0 & 95 \\
\hline $101-3$ & 0.5 & 0.3 & 1.0 & 5.3 & 0.0 & 95 \\
\hline
\end{tabular}

